\title{
New method of electron beam transverse size measurement by angular distribution of emission in a thin crystal
}

\author{
Yu. A. Goponov, ${ }^{1}$ S. A. Laktionova, ${ }^{1}$ R. A. Shatokhin, ${ }^{1}$ M. A. Sidnin, ${ }^{1}$ \\ K. Sumitani, ${ }^{2}$ Y. Takabayashi, ${ }^{3}$ and I. E. Vnukov ${ }^{1, *}$ \\ ${ }^{1}$ Belgorod National Research University, 85 Pobedy stroenie, 308015 Belgorod, Russia \\ ${ }_{2}^{2}$ Japan Synchrotron Radiation Research Institute (JASRI), 1-1-1 Kouto, Sayo-cho, \\ Sayo-gun, Hyogo 679-5198, Japan \\ ${ }^{3}$ SAGA Light Source, 8-7 Yayoigaoka, Tosu, Saga 841-0005, Japan
}

(Received 21 October 2018; published 26 August 2019)

\begin{abstract}
A technique is proposed for determining beam dimensions on a target by measuring two-dimensional angular distributions of the radiation for two distances between the crystal where the radiation is generated and a coordinate detector. The dimensions are determined from the results of a least squares method procedure with varying parameters, where the adjustable function is the distribution for a shorter distance and the fitting function is the convolution of the angular distribution at a greater distance with a twodimensional Gaussian distribution whose parameters are uniquely related to the beam dimensions on the target and the distances between the crystal and the detector. The minimum measured beam sizes are about 50-60 $\mu \mathrm{m}$ for the parametric x-ray mechanism and an electron energy of less than $1 \mathrm{GeV}$ and 10-15 $\mu \mathrm{m}$ for the mechanism of diffracted transition radiation and electrons with an energy above several GeV.
\end{abstract}

DOI: $10.1103 /$ PhysRevAccelBeams.22.082803

\section{INTRODUCTION}

The divergence and transverse dimensions of accelerated particles beams are important parameters of any accelerator. Therefore, many methods for measuring them have been developed and employed. Along with the use of fluorescent screens, optical transition radiation (OTR) and optical diffraction radiation are widely used to measure the electron beam parameters. However, it has recently been shown that OTR cannot be used to measure the profile of electron beams of linear accelerators used to create a freeelectron x-ray laser [1], since the OTR becomes coherent if the longitudinal size of the beam becomes comparable with the wavelength of the detected radiation [2].

The most natural method to avoid this coherence effect is to use radiation with a shorter wavelength. Several years ago $[3,4]$, the use of parametric $\mathrm{X}$-ray radiation (PXR) to determine the beam sizes was proposed. PXR is emitted in the Bragg direction when a fast charged particle moves through a crystal target; see, e.g., Ref. [5] and the literature cited there. The benefits of using PXR for measuring the particle beam parameters include good agreement between measurement results and calculations for a wide range of

\footnotetext{
*vnukov@bsu.edu.ru
}

Published by the American Physical Society under the terms of the Creative Commons Attribution 4.0 International license. Further distribution of this work must maintain attribution to the author(s) and the published article's title, journal citation, and DOI. electron and photon energies [6], a low background level, and the facility to change the photons' energy and their angular distribution by varying the orientation of the crystal.

Measurements of the PXR angular distributions for fast electrons in thin crystals with the help of coordinate detectors, reported in Refs. $[4,7,8]$, confirmed the possibility of determining the beam sizes using a detector located in the immediate vicinity of the crystal [4], changing the shape of the detected distributions depending on the beam size at the crystal [7], and determining the beam size of particles on a target with a pinhole camera [8].

Locating the coordinate detector in the immediate vicinity of the crystal [4] is not always possible. In addition, the problem arises of separating the detected radiation from the background, the source of which are the elements of the accelerator design. The use of a pinhole camera assumes an azimuthal symmetry of the angular distribution of the detected radiation; if this is not the case, an error will arise in determining the beam dimensions from the measurements [8].

Another significant disadvantage of the method in Ref. [8] is the duration of the measurement process, due to the hard collimation of the recorded radiation. These shortcomings require the development of new methods for measuring the transverse dimensions of a particle beam based on measuring the total angular distribution of the PXR and taking into account its features, in particular, the azimuthal asymmetry and the contributions of other coherent radiation mechanisms such as diffracted transition 
radiation (DTR) and diffracted bremsstrahlung (DB); see, e.g., Refs. $[6,9,10]$, and references therein.

From the foregoing, it is important and relevant to conduct an analysis of the dependence of the angular distributions of the coherent radiation of electrons in crystals from the beam dimensions, the observation angle, and other experimental conditions, aimed at developing a new technique for determining the transverse dimensions of electron beams of high energy.

Another method of beam size measurement is the use of so-called wire scanners [11-13]. These devices measure secondary electron emission [11] or bremsstrahlung, arising from the beam interaction with a thin carbon or tungsten wire moved across the beam $[12,13]$. The method allows the beam size to be determined up to 5-8 $\mu \mathrm{m}$. To determine the strengths and weaknesses of the suggested new method as an alternative to existing methods, it is instructive to compare it with the wire scanner method.

\section{ACCOUNTING FOR THE SPATIAL DIMENSIONS OF THE ELECTRON BEAM}

As noted above, an advantage of the coherent radiation of electrons in crystals for measuring the dimensions of an electron beam is the good agreement between measurement results and the calculations of Ref. [6], which allows us to analyze the dependence of the characteristics of the angular distribution of radiation on beam parameters through calculations.

The influence of the beam dimensions and the distance between the crystal and the detector on the measured twodimensional angular distribution of coherent radiation (see, e.g., Ref. [4]) can be described by the following expression:

$Y\left(\theta_{y}^{\prime}, \theta_{x}^{\prime}\right)=\iint Y\left(\theta_{y}, \theta_{x}\right) G\left(\theta_{y} \rightarrow \theta_{y}^{\prime}, \theta_{x} \rightarrow \theta_{x}^{\prime}\right) d \theta_{y} d \theta_{x}$,

where $\theta_{x}$ and $\theta_{y}$ are the horizontal and vertical projections, respectively, of the photon emission angle, measured relative to the Bragg direction. The reflecting plane is positioned vertically. The functions $Y\left(\theta_{y}^{\prime}, \theta_{x}^{\prime}\right)$ and $Y\left(\theta_{y}, \theta_{x}\right)$ are the angular distributions of the coherent radiation for an extended and pointlike beam of particles on the target, respectively. Integration is carried out within a full solid angle. The function $G\left(\theta_{y} \rightarrow \theta_{y}^{\prime}, \theta_{x} \rightarrow \theta_{x}^{\prime}\right)$ describes the relationship between the variables of each of these distributions. For a two-dimensional Gaussian distribution of the spread of the points of entry of electrons into a crystal, the function can be represented as

$$
\begin{aligned}
& G\left(\theta_{y} \rightarrow \theta_{y}^{\prime}, \theta_{x} \rightarrow \theta_{x}^{\prime}\right) \\
& =\frac{R^{2}}{2 \pi \sigma_{y} \sigma_{x}} \exp \left(\frac{-R^{2}\left(\theta_{y}-\theta_{y}^{\prime}\right)^{2}}{2 \sigma_{y}^{2}}\right) \exp \left(\frac{-R^{2}\left(\theta_{x}-\theta_{x}^{\prime}\right)^{2}}{2 \sigma_{x}^{2}}\right),
\end{aligned}
$$

where $\sigma_{x}$ and $\sigma_{y}$ are characteristic beam dimensions in the horizontal and vertical planes, respectively, and $R$ is the distance between the crystal and the detector.

In the experiment, the dependence of the output of the radiation incident on the detector with a fixed aperture on its position, that is, the vertical $Y\left(\theta_{y_{i}}\right)$ or horizontal $Y\left(\theta_{x_{i}}\right)$ angular distributions, is determined. In particular, the vertical distribution can be written in the following form:

$$
Y\left(\theta_{y_{i}}\right)=\iint_{\Delta \Omega\left(y_{i}\right)} Y\left(\theta_{y}^{\prime}, \theta_{x}^{\prime}\right) d \theta_{y}^{\prime} d \theta_{x}^{\prime},
$$

where $\Delta \Omega\left(y_{i}\right)$ is the solid angle overlapped by the detector located at the point $y_{i}$, over which the integration is carried out.

Using the calculation technique in Ref. [14] to account for the spatial distribution of electrons incident on the crystal requires taking into account the change in the angle of the detector relative to the direction of the electron beam for each point of the crystal on which the electrons fall. This requires considerable computational time, making the calculation almost impossible. However, the fact that a shift of the emitting point is nearly equivalent to a change in the position of the angle of the detector's location in the vertical or horizontal plane makes it possible to replace this shift by a corresponding displacement of the detector position. The characteristic size of the electron beam usually does not exceed several hundred microns, whereas the distance between the crystal and the detector is usually on the order of one meter. Therefore, the change in the solid angle covered by the detector unit due to a shift of the radiating point in the first approximation can be neglected.

In other words, the presence of a beam projection on the target is equivalent to increasing the real size of the detector $\pm \delta_{x} / 2, \pm \delta_{y} / 2$ in both planes to $\pm \Delta x / 2= \pm \delta_{x} / 2 \pm n \sigma_{x}$ and $\pm \Delta y / 2= \pm \delta_{y} / 2 \pm n \sigma_{y}$, where $\sigma_{x, y}$ is the characteristic size of the electron beam in the horizontal and vertical planes and $n$ is the number of standard deviations considered. Because of the difference in the physics of the process and the methodology for calculating the PXR and the diffraction of real photons in the method of Ref. [14], calculation methods that take into account the transverse dimensions of the electron beam on the target are different for each of these radiation mechanisms.

The method [14] is based on the PXR kinematic theory [15] and dynamic X-ray diffraction theory using the Darvin and Prins approach for x-ray multiple rediffraction on the crystal planes $[16,17]$. This allows the description of the absolute coherent emission yield and the emission angular distributions for a pointlike electron beam with an error of less than $15 \%$ [18].

A detailed description of the technique for each radiation mechanism and a comparison between experimental results $[19,20]$ and calculations is given in Ref. [21]. This technique was used to compare measured PXR spatial 
distributions from a thin silicon crystal and controlled sizes of an electron beam [7] with calculations and showed a satisfactory agreement [22].

\section{ANALYSIS OF DEPENDENCE OF RADIATION ANGULAR DISTRIBUTIONS ON BEAM SIZES}

The good agreement between the measured and calculated dependences obtained in Ref. [21] allows us to analyze the effect of the electron beam profile on the recorded radiation intensity distribution by calculations and to choose the most simple and convenient way of measuring the characteristic beam dimensions. To simplify the problem, the calculations are performed for the experimental conditions in Ref. [20] for the reflecting plane (011). The electron beam energy is $255 \mathrm{MeV}$, and the sample used is a diamond with a large face perpendicular to the $\langle 100\rangle$ axis and a thickness of $50 \mu \mathrm{m}$. The dimensions of the electron beam on the target are $\sigma_{x}=0.3 \mathrm{~mm}$ and $\sigma_{y}=0.9 \mathrm{~mm}$.

As can be seen from Eqs. (1) and (2), a change in the distance between the crystal and the detector leads to a change in the recorded radiation distribution, which can be used to estimate the beam size. To demonstrate this, Fig. 1 shows the vertical angular distributions of radiation for the following distances $R$ between the crystal and the detector: $5,1,0.5$, and $0.2 \mathrm{~m}$. All the conditions except for the distances coincide with the conditions of the experiment in Ref. [20]. For convenience of comparison, the calculation is performed for different detector element sizes and motion steps, chosen so that the solid angle overlapped by the detector unit remains constant. Since the photon yield for higher reflection orders does not exceed 3\%-5\% of the first allowed order intensity of reflection (022) [20], the contributions of higher orders were not taken into account.

As can be seen from Fig. 1, the distribution for the distance of $5 \mathrm{~m}$ practically coincides with that for a pointlike beam. As $R$ decreases, the difference between

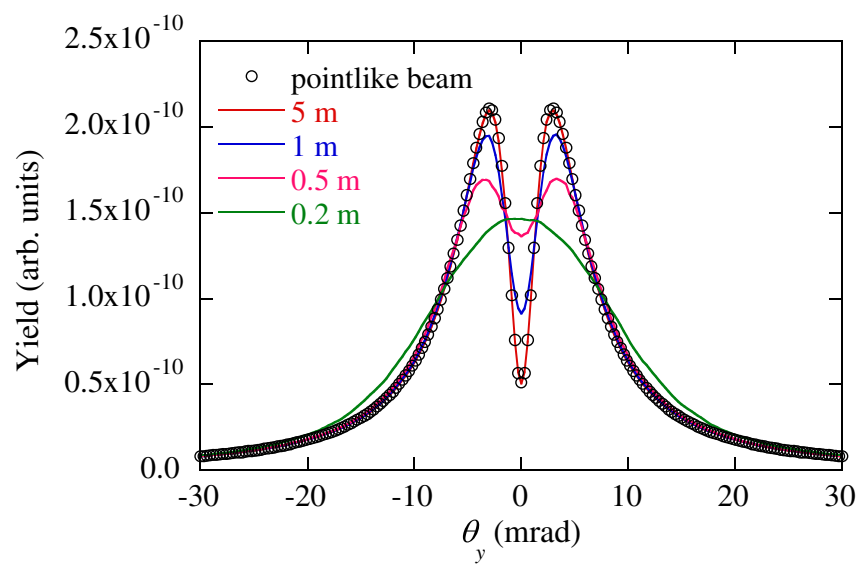

FIG. 1. Vertical angular distribution of radiation for the experimental conditions in Ref. [20] and the reflecting plane (011). Electron energy $255 \mathrm{MeV}$; diamond crystal thickness, $50 \mu \mathrm{m}$; (022) reflection order; observation angle $\Theta_{D}=32.2^{\circ}$. these distributions and the distribution for a pointlike beam increases.

As a characteristic of the shape of the measured angular distribution of the radiation yield $Y$ for a fixed distance between the crystal and the detector $R_{j}$, it is most convenient to take the variance of this distribution $D\left(\theta_{y}\right)_{R_{j}}$ :

$$
D\left(\theta_{y}\right)_{R_{j}}=\frac{\sum_{i=1}^{n} Y_{R_{j}}\left(\theta_{y_{i}}\right) \cdot\left(\theta_{y_{i}}-\bar{\theta}_{R_{j}}\right)^{2}}{\sum_{i=1}^{n} Y_{R_{j}}\left(\theta_{y_{i}}\right)},
$$

where $n$ is the number of points in the measured dependence and $\bar{\theta}_{R_{j}}$ is the coordinates of its center of gravity.

The simulation shows that the distribution variance increases when the beam size increases and when the distance between the crystal and detector decreases. To confirm this, Fig. 2 presents the dependence of the effective divergence $\sigma_{y_{j}}^{\prime}=\sqrt{D\left(\theta_{y}\right)_{R_{j}}-D\left(\theta_{y}\right)_{R=\infty}}$ on the distance between the crystal and the detector for the conditions of the experiment [20]. The electron beam sizes are $\sigma_{x}=$ $0.3 \mathrm{~mm}$ and $\sigma_{y}=0.9 \mathrm{~mm}$. Here, $D\left(\theta_{y}\right)_{R_{j}}$ and $D\left(\theta_{y}\right)_{R=\infty}$ are the variances of the radiation angular distributions for extended and pointlike electron beams, respectively (see Fig. 1).

Figure 2 shows that $\sigma_{y_{j}}=R_{j} \cdot \sigma_{y_{j}}^{\prime}$ changes from 0.75 to $0.9 \mathrm{~mm}$, has a weak dependence on the distance between the crystal and the detector, and is close to the beam size value $\sigma_{y}=0.9 \mathrm{~mm}$ used in the simulation. A significant difference is observed only for $R_{j}=5 \mathrm{~m}$, where the emission angular distribution practically coincides with that for a pointlike beam and $\sigma_{y_{j}}^{\prime^{2}} \ll D\left(\theta_{y}\right)_{R_{j}}$.

As noted above, a similar ratio of beam dimensions to the target measured with the aid of PXR detection and optical transition radiation was obtained in Ref. [8]. Therefore, $R_{j} \cdot \sigma_{y_{j}}^{\prime}$ can be considered an estimate of the beam size $\tilde{\sigma_{y}}$. The observed difference between $\tilde{\sigma_{y}}$ and $\sigma_{y}$ is due to the

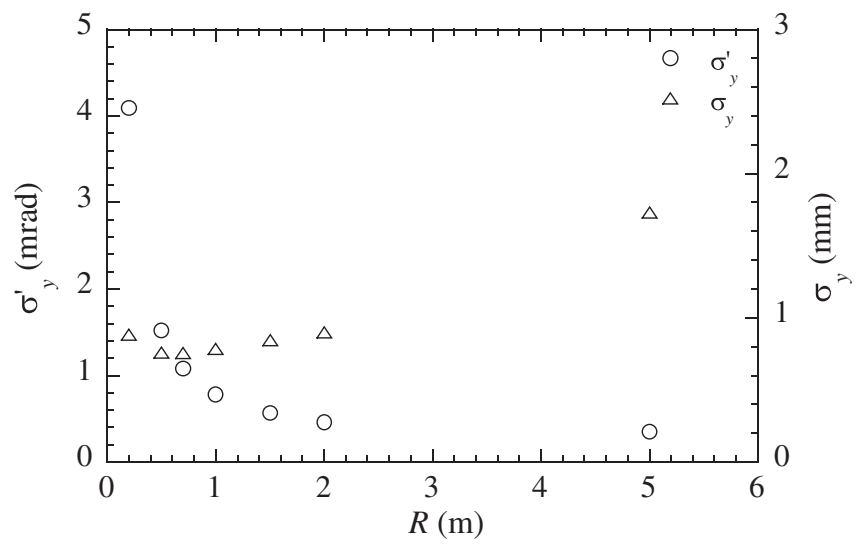

FIG. 2. Dependence of the effective divergence $\sigma_{y}^{\prime}$ and the beam size estimate $\sigma_{y}$ on the distance between the crystal and the detector for the conditions of the experiment [20]. 
influence of the beam dimensions in the horizontal plane on the measured vertical distribution. The simulation shows that, for the horizontal beam size $\sigma_{x} \sim 0.01 \mathrm{~mm}$, the values $\tilde{\sigma_{y}}$ and $\sigma_{y}$ coincide.

The main difficulty in using the proposed technique in a real experiment is the requirement to have information on the variance of the angular distribution for a pointlike beam or the angular distribution measured for a very large distance between the crystal and the detector. The use of the calculated distribution in practice is not feasible, since the efficiency of coordinate detectors is known with an accuracy not better than 5\%-10\%. Moreover, the divergence of the electron beam and the degree of crystal imperfection, which affect the angular distribution of radiation, as a rule are not known precisely.

To solve this problem, we can use the fact that the unknown variance of the distribution for a pointlike beam in accordance with the expression $\sigma_{y_{j}}^{\prime}=$ $\sqrt{D\left(\theta_{y}\right)_{R_{j}}-D\left(\theta_{y}\right)_{R=\infty}}$ can be obtained from measurements of another distance between the crystal and the detector, which allows us to neglect the value $D\left(\theta_{y}\right)_{R=\infty}$.

Consider measurements carried out for two distances between the crystal and the detector $R_{1}$ and $R_{2}$, and $R_{1}=k \cdot R_{2}$, where $k$ significantly differs from unity. In this case, we can write the equation relating the variance of the distribution for these distances:

$$
\left(\sigma_{y} / R_{2}\right)^{2}-\left(\sigma_{y} / R_{1}\right)^{2} \approx D\left(\theta_{y}\right)_{R_{2}}-D\left(\theta_{y}\right)_{R_{1}} .
$$

From this, we can obtain the following estimate of the characteristic beam size:

$$
\widetilde{\sigma_{y}} \approx \frac{k \cdot R_{2}}{\sqrt{k^{2}-1}} \sqrt{D\left(\theta_{y}\right)_{R_{2}}-D\left(\theta_{y}\right)_{R_{1}}} .
$$

The estimate obtained in this way using dependencies in Fig. 1 for distances between the crystal and detector of $R_{1}=1 \mathrm{~m}$ and $R_{2}=0.5 \mathrm{~m}$ gives $\tilde{\sigma_{y}} \approx 0.78 \mathrm{~mm}$, which coincides with the value obtained using the angular distribution for the pointlike beam and the distance of $1 \mathrm{~m}$ $\sigma_{y}=0.784 \mathrm{~mm}$; see Fig. 2 .

Calculations show that the beam size on the target also affects the angular distribution for other orientations, crystals, and observation angles. The degree of this influence depends on the ratio of the value $\sigma^{\prime}=\sigma / R$ and the characteristic angle of photon emission at $\Theta_{\mathrm{ph}}=\sqrt{\gamma^{-2}+\frac{\omega_{p}^{2}}{\omega^{2}}}$, where $\gamma$ is the Lorentz factor and $\omega$ and $\omega_{p}$ are the photon energy and the plasmon energy of the medium, respectively.

For large $\Theta_{\mathrm{ph}}$ and small $\sigma^{\prime}$, the difference between $D\left(\theta_{y}\right)_{R_{j}}$ and $D\left(\theta_{y}\right)_{R=\infty}$ appears too small, and the equations $\sigma_{y_{j}}^{\prime}=\sqrt{D\left(\theta_{y}\right)_{R_{j}}-D\left(\theta_{y}\right)_{R=\infty}}$ and $\widetilde{\sigma_{y_{j}}}=R_{j} \cdot \sigma_{y_{j}}^{\prime}$ become invalid. The distance of $5 \mathrm{~m}$ where $\tilde{\sigma_{y}} \sim 1.7 \mathrm{~mm}$ is about twice the real beam size (see Fig. 2) and corresponds with a ratio of $\sigma^{\prime}=0.18 \mathrm{mrad}$ and $\Theta_{\mathrm{ph}}=$ $2.94 \mathrm{mrad}$ of about 0.06 . Therefore, confident use of the proposed method for estimating the size of the beam on the target $\sigma$ is possible only if the condition $\sigma^{\prime}=\sigma / R_{2} \geq$ $0.1 \Theta_{\text {ph }}$ is satisfied, where $R_{2}$ is the smaller of the two distances between the crystal and the detector.

The main problem in its realization is the ineradicable mutual influence of the beam sizes in the vertical and horizontal planes on the recorded angular distributions in another direction, as noted above.

\section{PROPOSED MEASUREMENT TECHNIQUE}

It is not possible to solve the problem of the mutual influence of the beam sizes in both planes on the measured radiation intensity distribution in these directions. Therefore, from the one-dimensional distributions described in formula (3) in the vertical and horizontal directions, it is necessary to go to two-dimensional distributions. For a coordinate detector located at a distance $R$, the measured two-dimensional radiation intensity distribution $Y_{R}\left(\theta_{y_{i}}, \theta_{x_{j}}\right)$ can be written in analogy with Eq. (3) in the following form:

$$
Y_{R}\left(\theta_{y_{i}}, \theta_{x_{j}}\right)=\iint_{\Delta \Omega\left(y_{i}, x_{j}\right)} Y_{R}\left(\theta_{y}^{\prime}, \theta_{x}^{\prime}\right) d \theta_{y}^{\prime} d \theta_{x}^{\prime},
$$

where $Y_{R}\left(\theta_{y}^{\prime}, \theta_{x}^{\prime}\right)$ is the convolution of the radiation angular distribution for the pointlike beam with a Gaussian distribution whose parameters, in accordance with Eqs. (1) and (2), are determined by the beam sizes and the distance between the crystal and the detector. As before, $\Delta \Omega\left(y_{i}, x_{j}\right)$ is the solid angle overlapped by the detector element located at the point $y_{i}, x_{j}$, over which the integration is performed.

The difference between the distributions $Y_{R_{1}}\left(\theta_{y_{i}}, \theta_{x_{j}}\right)$ and $Y_{R_{2}}\left(\theta_{y_{i}}, \theta_{x_{j}}\right)$, measured for distances $R_{1}$ and $R_{2}$, respectively, is due only to the characteristic beam dimensions and the distances between the crystal and the detector. As in the case of one-dimensional distributions, we assume that $R_{1}=k \cdot R_{2}$ and the solid angles overlapped by the detector units are the same in each measurement. In the first approximation, we can assume that $Y_{R_{2}}\left(\theta_{y_{i}}, \theta_{x_{j}}\right)$ is the convolution of the distribution $Y_{R_{1}}\left(\theta_{y_{i}}, \theta_{x_{j}}\right)$ with a Gaussian distribution with a variance that depends on the unknown sizes of the beam on the target and the values $R_{1}$ and $R_{2}$.

To determine the beam dimensions on the target, we use the least squares method, minimizing the quadratic form:

$$
\begin{aligned}
& \sum_{i=1}^{n} \sum_{j=1}^{m}\left[Y_{R_{2}}\left(\theta_{y_{i}}, \theta_{x_{j}}\right)-\frac{1}{2 \pi \sigma_{x}^{\prime} \sigma_{y}^{\prime}} \sum_{i^{\prime}=1}^{n} \sum_{j^{\prime}=1}^{m} Y_{R_{1}}\left(\theta_{y_{i^{\prime}}}, \theta_{x_{j^{\prime}}}\right)\right. \\
& \left.\times \exp \left(-\frac{\left(\theta_{y_{i}}-\theta_{y_{i^{\prime}}}\right)^{2}}{2\left(\sigma_{y}^{\prime}\right)^{2}}\right) \exp \left(-\frac{\left(\theta_{x_{j}}-\theta_{x_{j^{\prime}}}\right)^{2}}{2\left(\sigma_{x}^{\prime}\right)^{2}}\right)\right]^{2}=\operatorname{Min},
\end{aligned}
$$


where $m$ and $n$ are the number of points of the measured distributions in the horizontal and vertical directions, respectively. By analogy with the one-dimensional case, the fitting parameters $\sigma_{x}^{\prime}$ and $\sigma_{y}^{\prime}$, minimizing this form, are related to the beam size estimates on the target $\widetilde{\sigma_{x}}$ and $\widetilde{\sigma_{y}}$ as follows:

$$
\tilde{\sigma}_{y, x} \approx \frac{k \cdot R_{2}}{\sqrt{k^{2}-1}} \sigma_{y, x}^{\prime}
$$

To test the proposed method and determine the limits of its applicability, a simulation of the beam size estimation from two-dimensional distributions using the convolution parameter variation method is carried out. The simulation is performed for the experimental conditions in Ref. [19], that is, a silicon crystal with a (011) reflecting plane and an observation angle of $32.2^{\circ}$. The characteristic angle of the PXR emission is $\Theta_{\mathrm{ph}}=3.317 \mathrm{mrad}$. The electron energy is $E_{0}=255 \mathrm{MeV}$. The detector pixel sizes are 0.1 and $0.2 \mathrm{~mm}$ in both directions for $R_{2}=0.5 \mathrm{~m}$ and $R_{1}=1 \mathrm{~m}$, respectively. The sizes of the electron beam on the crystal are $\sigma_{x}=0.3 \mathrm{~mm}$ and $\sigma_{y}=0.8 \mathrm{~mm}$. Figure 3 shows the vertical (a) and horizontal (b) distributions passing through the reflex center. As the model angular distribution of the PXR, the Feranchuk-Ivashin distribution [23] is chosen:

$$
Y_{\mathrm{PXR}}\left(\theta_{x}, \theta_{y}\right)=N_{\mathrm{PXR}}(\omega) \frac{\theta_{x}^{2} \cos ^{2} 2 \Theta_{B}+\theta_{y}^{2}}{\left(\theta_{x}^{2}+\theta_{y}^{2}+\Theta_{\mathrm{ph}}^{2}\right)^{2}}
$$

Here, $N_{\text {PXR }}(\omega)$ is the multiplier that characterizes the PXR yield, and $\Theta_{B}$ is the angle of the crystal plane rotation with respect to the electron beam direction.

To obtain the radiation angular distribution for a pointlike beam, the model distribution was convolved with a two-dimensional Gaussian distribution with $\theta_{e}=0.3 \mathrm{mrad}$, where $\theta_{e}$ is the electron beam divergence. The contribution of the diffraction of real photons to the total radiation intensity for these conditions does not exceed a few percent [14] and, therefore, is not taken into account.

Two other curves are shown in Fig. 3, corresponding to the extended electron beam for distances between the crystal and detector of $R_{1}=2 R_{2}=1 \mathrm{~m}$ and $R_{2}=0.5 \mathrm{~m}$, respectively. To take into account the possible effect of the statistical spread of the measurement results, the dependences for the extended electron beams are made to be "noisy" according to the uniform distribution and error region $\pm 10 \%$ at each point.

The error in determining the fitting parameters and estimating the beam dimensions obtained using the proposed technique does not exceed a few percent. The dependence obtained by "fitting" is practically the same as the curve for the shorter distance and, therefore, is not given.

To determine the method sensitivity, we carried out a sequence of beam size estimations for the same beam size values and different noisy angular distributions from the
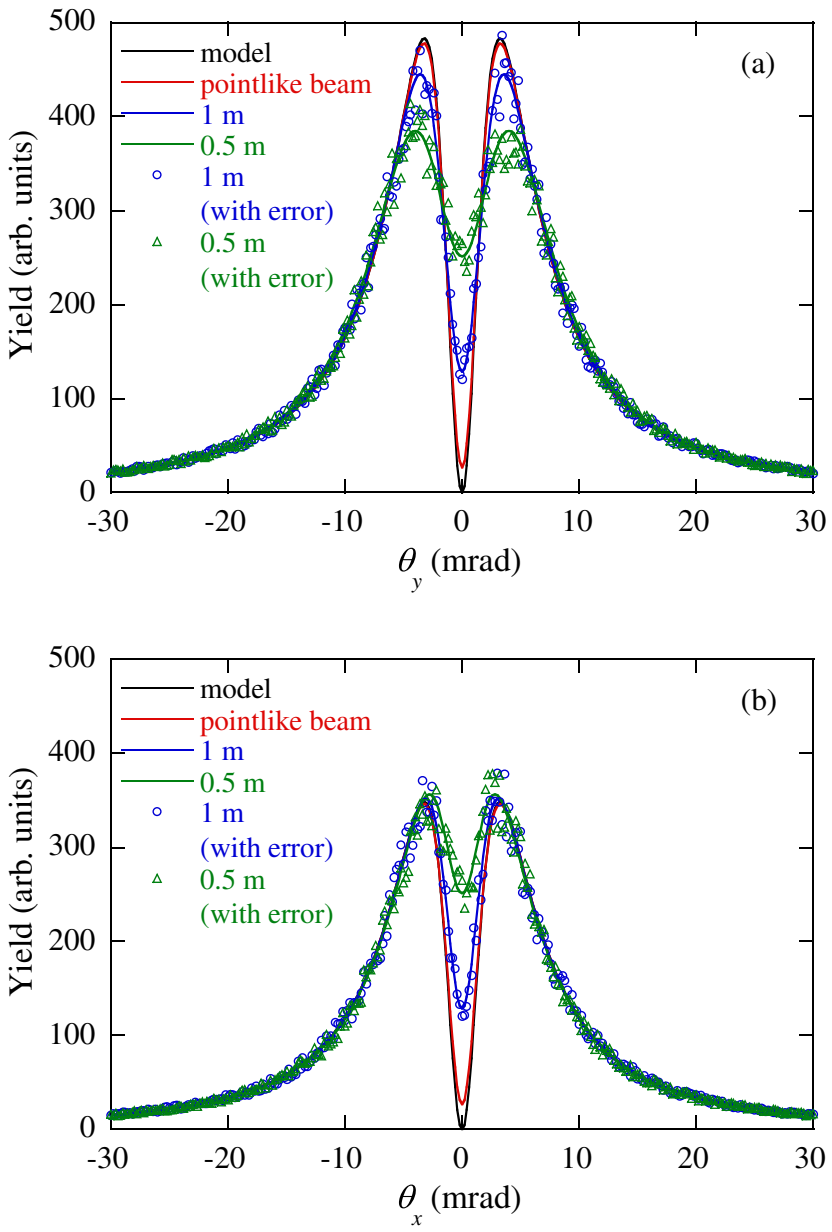

FIG. 3. Angular distribution of radiation in the vertical (a) and horizontal (b) directions, showing a model PXR angular distribution, radiation distribution for a pointlike electron beam, and distributions for the extended electron beam with distances $R_{1}=$ $1 \mathrm{~m}$ and $R_{2}=0.5 \mathrm{~m}$. Electrons energy $255 \mathrm{MeV}$; silicon crystal; (022) reflection order; observation angle $\Theta_{D}=32.2^{\circ}$.

distances between the crystal and the detector. The simulations were made for $\sigma_{x}=0.3 \mathrm{~mm}$ and $\sigma_{y}=0.8 \mathrm{~mm}$. The other parameters were the same as for Fig. 3. The dependence of the obtained $\tilde{\sigma}_{y, x}$ values on the shorter distance is presented in Fig. 4. As errors, we took the standard deviations of the estimated values from the average value. As before, the ratio $R_{1}=2 R_{2}$ is fulfilled.

From Fig. 4, we can see that for this distance region the errors of the beam size estimate in the vertical and horizontal directions are not worse than 5\%-7\%. However, for a distance of more than $1 \mathrm{~m}$, the $\tilde{\sigma}_{x}$ values differ from the true amount $\sigma_{x}=0.3 \mathrm{~mm}$, while the $\tilde{\sigma}_{y}$ values are close to the real amount $\sigma_{y}=0.8 \mathrm{~mm}$. For these distances, the ratio of $\sigma_{x}^{\prime}=\sigma_{x} / R_{2}<0.3 \mathrm{mrad}$ and $\Theta_{\mathrm{ph}}=$ $3.317 \mathrm{mrad}$ becomes less than 0.1 . For both the onedimensional and two-dimensional cases for smaller values of $\sigma^{\prime}$, the distinction between distributions for different distances is practically absent, and the method loses sensitivity. 


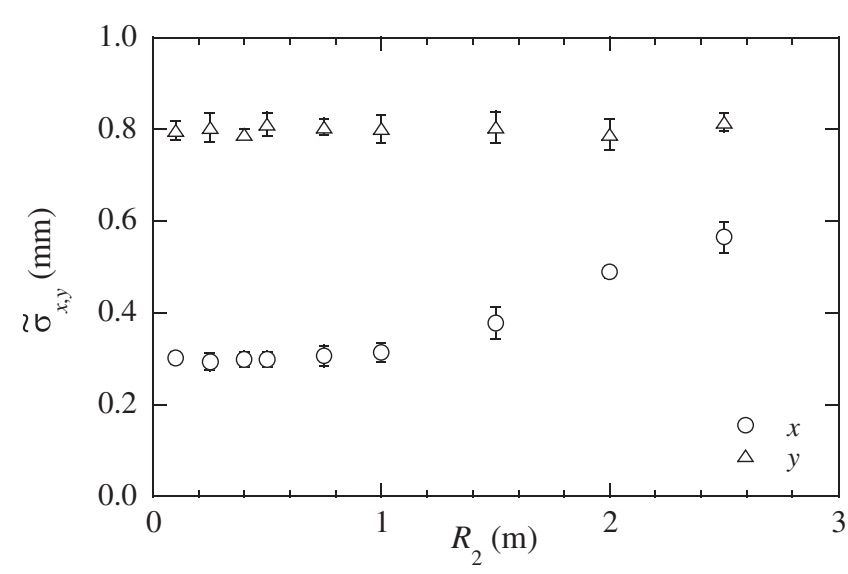

FIG. 4. Dependence of the beam size estimates from the distance between the crystal and the detector. Silicon; electron energy $255 \mathrm{MeV}$; observation angle $\Theta_{D}=32.2^{\circ} ; \sigma_{x}=0.3 \mathrm{~mm}$; $\sigma_{y}=0.8 \mathrm{~mm}$.

It is important to note that the two-dimensional method allows us to determine the beam size in both directions, while the one-dimensional case provides an estimate of the larger beam size only.

Equally important is the requirement $\sigma_{x, y} \sim \delta_{x, y}$, that is, the size of the element of the coordinate detector $\delta_{x, y}$ should be comparable with the characteristic particle beam dimensions on the target. For a pixel size in both directions of $0.05 \mathrm{~mm}, \tilde{\sigma}_{y, x}$ can be reliably determined with an error of about $10 \%$ even for $R_{2}=0.5 \mathrm{~m}$ and beam sizes $\sigma_{x}=$ $0.1 \mathrm{~mm}$ and $\sigma_{y}=0.3 \mathrm{~mm}$, that is, for $\sigma_{x}^{\prime} \sim 0.05 \Theta_{\mathrm{ph}}$.

Assuming that the observation angle and the distance between the crystal and the coordinate detector, achieved in the experiment [7], are close to the limits, it is possible to estimate the minimum sizes of the electron beam available for the proposed method. This experiment was performed for an electron energy of $855 \mathrm{MeV}$, (004) reflection order of a silicon crystal, an observation angle of $22.5^{\circ}$, and a distance between the crystal and the detector of $R=0.35 \mathrm{~m}$ that gave a spacing between the electron beam line and the detector center of about $130 \mathrm{~mm}$. The characteristic angle of the PXR emission is $\Theta_{\mathrm{ph}} \approx 1.54 \mathrm{mrad}$, and the minimum confidently estimated beam size is $\sigma \approx 0.1 R_{2} \cdot \Theta_{\mathrm{ph}} \sim 50-60 \mu \mathrm{m}$.

In other words, a technique based on measuring the spatial distribution of the PXR beam for two distances provides a beam size measurement of the order of $100 \mu \mathrm{m}$ and higher, which is typical for middle energy accelerators. However, this does not make possible measurements on the order of tens of microns in modern sources of fourthgeneration radiation [1], for which there is a problem in measuring the transverse sizes of an electron bunch with small longitudinal sizes [2].

As noted in Ref. [24] and confirmed in Refs. [25,26], for an electron energy above several $\mathrm{GeV}$, the contribution of the DTR at the center of the reflex becomes significantly

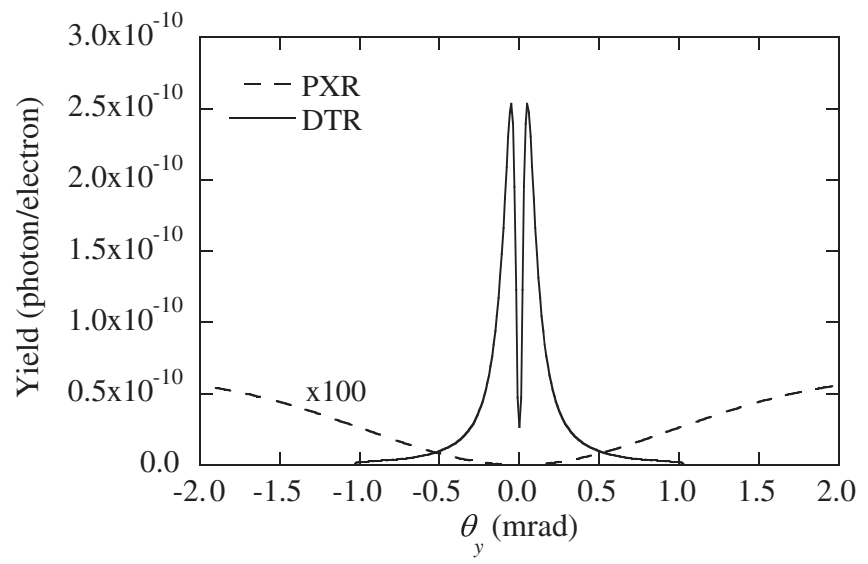

FIG. 5. Vertical angular distribution of radiation for reflection (022) from a silicon crystal, electron energy $10 \mathrm{GeV}$, and observation angle $\Theta_{D}=32.2^{\circ}$.

greater than the contribution of the PXR, and the resulting angular distribution of the radiation yield varies significantly. Figure 5 gives an example vertical angular distribution of the radiation for a pointlike electron beam and an observation angle $\Theta_{D}=32.2^{\circ}$, an electron energy of $10 \mathrm{GeV}$, and a silicon crystal $20 \mu \mathrm{m}$ thick with the plane (011) turned on an angle $\Theta_{B}=16.1^{\circ}$. The divergence of the electron beam is $5 \mu \mathrm{rad}$. Located at a distance of $2 \mathrm{~m}$ from the crystal, a detector with dimensions of $20 \times 20 \mu \mathrm{m}^{2}$ is translated in steps of $20 \mu \mathrm{m}$ through the center of the radiation angular distribution. The curves are the results of calculating PXR and DTR using the technique of Ref. [14]. The contribution of DB is absent, since $\omega=11.64 \mathrm{keV} \ll \gamma \omega_{p} \sim 603 \mathrm{keV}$.

As can be seen from Fig. 5, the angular density of the DTR is more than 500 times larger than the PXR intensity. In the center of the reflex, the difference is even greater. Therefore, the PXR contribution, in the first approximation, can be considered as having no singularities and can, in general, be neglected. The presence of a bright narrow peak in the angular distribution of the radiation close to the PXR angular distribution allows the use of the above-described technique not only for PXR, but also for DTR. In this case, the characteristic angle of photon output $\Theta_{\text {ch }}$ is close to $\gamma^{-1}$; that is, for electron energies above $5 \mathrm{GeV}$, it decreases by 10 or more times compared with $\Theta_{\mathrm{ph}}$. As a result, the minimum beam size measured by the proposed method decreases from hundreds to tens of microns.

To verify this, a simulation of the electron beam size estimate with an energy of $10 \mathrm{GeV}$ is carried out. The simulation is performed for the following conditions: reflection (022) from a silicon crystal, an observation angle of $32.2^{\circ}$, and detector element dimensions for a shorter distance of $0.01 \times 0.01 \mathrm{~mm}^{2}$. The dimensions of the electron beam on the crystal are $\sigma_{x}=15$ and $\sigma_{y}=20 \mu \mathrm{m}$. The divergence of the electron beam is $\theta_{e}=15 \mu \mathrm{rad}$. Figure 6 shows the DTR vertical angular distribution passing through the center 


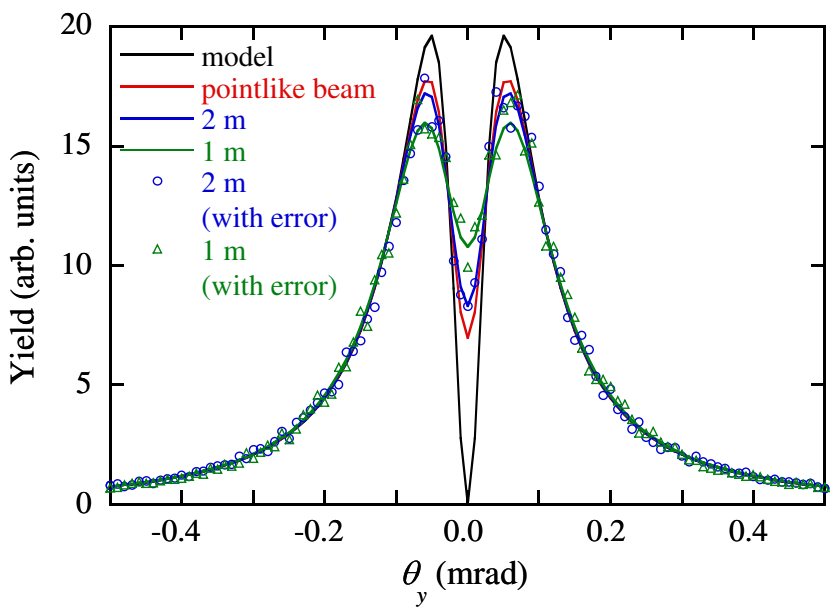

FIG. 6. Vertical angular distribution of radiation. Model DTR angular distribution, radiation distribution for a pointlike electron beam, and distributions for the extended electron beam and distances $R_{1}=2 \mathrm{~m}$ and $R_{2}=1 \mathrm{~m}$. Electron energy $10 \mathrm{GeV}$; silicon crystal; (022) reflection order; observation angle $\Theta_{D}=32.2^{\circ}$.

of the radiation spot. As has been shown in several papers (see, e.g., Refs. [10,27]), the two-dimensional angular distribution of the DTR can be represented as

$Y_{\mathrm{DTR}}\left(\theta_{x}, \theta_{y}\right)=N_{\mathrm{DTR}}(\omega) \frac{\theta_{x}^{2} \cos ^{2} 2 \Theta_{B}+\theta_{y}^{2}}{\left[\left(\theta^{2}+\Theta_{\mathrm{ph}}^{2}\right)\left(\theta^{2}+\gamma^{-2}\right)\right]^{2}}$,

where $\theta^{2}=\theta_{x}^{2}+\theta_{y}^{2}$ and $N_{\mathrm{DTR}}(\omega)$ is the multiplier that characterizes the DTR output and depends on the viewing angle, the order of reflection, and the photon energy. For the reasons mentioned above, the PXR contribution was not taken into account. To obtain the radiation angular distribution for a pointlike electron beam, the DTR distribution was convolved with a two-dimensional Gaussian distribution with $\theta_{e}=15 \mu \mathrm{rad}$. Two other curves correspond to the extended electron beam and distances between the crystal and the detector of $R_{1}=2 \mathrm{~m}$ and $R_{2}=1 \mathrm{~m}$, respectively. To take into account the possible effect of the statistical spread of the measurement results, the dependences for an extended electron beam are made to be noisy at each point, similar to the PXR angular distribution (see the comments for Fig. 3).

As in the case of PXR, the error in determining the fitting parameters and estimating the beam dimensions obtained using the proposed technique for these conditions does not exceed a few percent.

To determine the method sensitivity, we carried out a sequence of beam size estimations for the same beam size and different noisy angular distributions from the distances between the crystal and the detector. The dependence of the obtained $\tilde{\sigma}_{y, x}$ values with their errors on the shorter distance is presented in Fig. 7. As before, the condition $R_{1}=2 R_{2}$ is fulfilled. The simulation was made for the beam sizes $\sigma_{x}=20 \mu \mathrm{m}$ and $\sigma_{y}=30 \mu \mathrm{m}$.

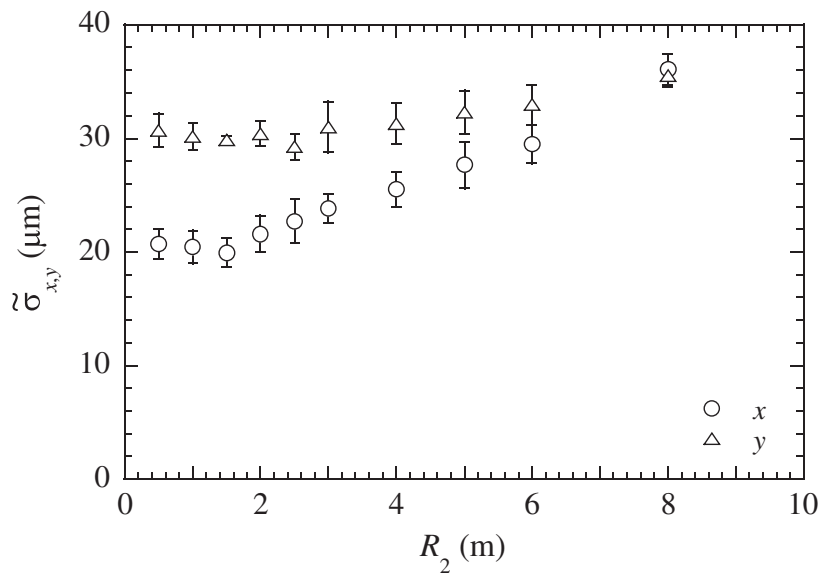

FIG. 7. Dependence of the beam size estimates on the distance between the crystal and the detector. Silicon; electron energy $10 \mathrm{GeV} ;$ observation angle $\Theta_{D}=32.2^{\circ} ; \quad \sigma_{x}=20 \mu \mathrm{m}$; $\sigma_{y}=30 \mu \mathrm{m}$.

From Fig. 7, we can see that for this distance region errors of the beam size estimate in the vertical and horizontal directions are no more than 5\%-7\%. As in the case of PXR, the difference between the estimated value $\tilde{\sigma}_{x, y}$ and the true amount $\sigma_{x, y}$ increases with increasing $R_{2}$. These differences become greater than the standard deviation values of $\tilde{\sigma}_{x, y}$ for $R_{2}$ of about 2.5 and $5 \mathrm{~m}$ for the horizontal and the vertical sizes, respectively, when $\sigma_{x, y}^{\prime}=\sigma_{x, y} / R_{2} \sim 0.1 \Theta_{\text {ch }}$ and less, where $\Theta_{\text {ch }}$ is $\gamma^{-1} \approx 51.1 \mu \mathrm{rad}$. For small values of $\sigma^{\prime}$, the distinction between distributions for different distances is practically absent, and the method loses sensitivity. It should be noted that for a larger "noise" level, a difference between the $\tilde{\sigma}_{x, y}$ value and true $\sigma_{x, y}$ amount begins to appear for smaller distances.

If the electron beam symmetry planes do not coincide with the horizontal and vertical planes (see, e.g., Ref. [13]), the azimuth direction angle between the symmetry and horizontal planes may also be included and determined under the fitting procedure.

The requirement of $\sigma_{x, y} \sim \delta_{x, y}$ in the case of DTR and electron energies above $5 \mathrm{GeV}$ becomes even more significant due to the limitation of the pixel size of $\mathrm{x}$-ray coordinate detectors. In the already mentioned work [7], a detector was used with pixel sizes of $11.2 \times 11.6 \mu \mathrm{m}^{2}$. Therefore, we can assume that the minimum confidently estimated beam size is $\sigma \geq 10 \mu \mathrm{m}$.

The main requirement for the successful implementation of the proposed method for estimating the dimensions of an electron beam on a target from measured radiation intensity distributions is the equality of the solid angles overlapped by the "element" of the detector for both distances. The emission of secondary electrons and photons from the region where $\mathrm{x}$-ray photons interact with the detector material distorts the measured distribution, "smoothing" it. This problem requires additional study, including the selection of a coordinate detector for possible 
measurements and determining an appropriate viewing angle, crystal, a model for the characteristics of the detector, and their effect on the measurement results.

\section{CONCLUSIONS}

The transverse size of a particle beam $\sigma_{x, y}$ in a linear electron accelerator can be determined by measuring the two-dimensional angular distributions of the particle radiation in thin crystals: PXR or DTR for two distinct distances between the crystal and the coordinate detector. As discussed in Sec. III, the horizontal beam size measurement is affected by the vertical beam size and vice versa for the one-dimensional method; therefore, we have proposed a two-dimensional method in Sec. IV, where the horizontal and vertical beam sizes can be obtained independently.

The PXR and DTR angular distributions look like a funnel with a solution angle $\Theta_{\mathrm{ch}}$. Therefore, in the angular distribution, the influence of the vertical beam size on the vertical angular distribution is stronger than the horizontal one and vice versa. This allows estimating both vertical and horizontal beam sizes almost separately.

The beam dimensions are determined by fitting the distribution for a shorter distance by a convolution of the distribution for a larger distance with a two-dimensional Gaussian distribution whose parameters are uniquely related to the beam dimensions and the distances between the crystal and the detectors.

The limit of the applicability of the technique is the condition $\sigma_{x, y} / R>0.1 \Theta_{\mathrm{ch}}$. For the PXR mechanism, the characteristic angle $\Theta_{\mathrm{ch}}$ coincides with $\Theta_{\mathrm{ph}}$, and, for the mechanism of DTR, for thin crystals and electron energies above several $\mathrm{GeV}$, it is close to $\gamma^{-1}$.

An additional requirement is the fulfillment of a condition on the ratio of the characteristic beam dimensions and the dimensions of the detector element $\sigma_{x, y} \sim \delta_{x, y}$. Simultaneous fulfillment of these requirements limits the measured beam size to 50-60 $\mu \mathrm{m}$ for the PXR mechanism and an electron energy of less than $1 \mathrm{GeV}$ and $10-15 \mu \mathrm{m}$ for the mechanism of DTR and electrons with an energy above several GeV.

The proposed method for estimating the electron beam sizes is model independent and does not require knowledge of the divergence of the beam and the degree of perfection of the crystal. The main requirement of the applicability of the technique is identifying the true angular distribution of the radiation in each of the measurements for different distances between the crystal and the detector and an adequate definition of the background, including for large observation angles relative to the center of the reflex, since the fit includes an allowance for the deviation of the distributions over the entire measured angular range. The technique is weakly sensitive to pulse heating of the target if it does not destroy the crystal [28] and can be used on intense beams of linear accelerators creating x-ray freeelectron lasers [1].

The best resolution of wire scanners (less than $10 \mu \mathrm{m}$ ) is better than that for the methods proposed in this manuscript. However, many conventional wire scanners employ wires with diameters of $100 \mu \mathrm{m}$ or less. These resolutions are comparable to that for our method. Furthermore, wire scanners have some disadvantages such as (i) some of them are one-dimensional monitors-i.e., the horizontal and vertical beam sizes cannot both be determined at the same time; (ii) wire vibration problems, which are severe especially for thin wires-i.e., high-resolution wire scanners; (iii) the handling of secondary radiation or particle detectors is not simple (sometimes careful simulation is necessary); and (iv) a bending magnet is necessary to measure bremsstrahlung beam cleaning.

In our proposed method, both the horizontal and vertical beam sizes can be determined at the same time. Moreover, $\mathrm{x}$ rays can be extracted to air through a Be window at a large angle with respect to the beam direction (i.e., the background level is low), and conventional two-dimensional $\mathrm{x}$-ray detectors can be used. We think that the experimental setup is no more complicated than that for wire scanners. Therefore, we believe that our proposed method is important as an alternative to wire scanners.

\section{ACKNOWLEDGMENTS}

This work was supported in part by JSPS KAKENHI Grant No. JP26400304.

[1] European X-Ray Free-Electron Laser Technical Design Report No. DESY 2006-097, 2007.

[2] H. Loos et al., in Proceedings of the 30th Free Electron Laser Conference (FEL08), Gyeongju, Korea (CERN, Geneva, 2008), p. 485.

[3] A. Gogolev, A. Potylitsyn, and G. Kube, A possibility of transverse beam size diagnostics using parametric X-ray radiation, J. Phys. Conf. Ser. 357, 012018 (2012).

[4] Y. Takabayashi, Parametric X-ray radiation as a beam size monitor, Phys. Lett. A 376, 2408 (2012).

[5] P. Rullhusen, X. Artru, and P. Dhez, Novel Radiation Sources Using Relativistic Electrons (World Scientific, Singapore, 1999).

[6] K.-H. Brenzinger et al., Investigation of the production mechanism of parametric X-ray radiation, Z. Phys. A 358, 107 (1997).

[7] G. Kube, C. Behrens, A. S. Gogolev, Yu. P. Popov, A. P. Potylitsyn, W. Lauth, and S. Weisse, in Proceedings of the 4th International Particle Accelerator Conference, IPAC2013, Shanghai, China, 2013 (JACoW, Shanghai, China, 2013), p. 491.

[8] Y. Takabayashi and K. Sumitani, New method for measuring beam profiles using a parametric X-ray pinhole camera, Phys. Lett. A 377, 2577 (2013). 
[9] E. A. Bogomazova, B. N. Kalinin, G. A. Naumenko, D. V. Padalko, A. P. Potylitsyn, A. F. Sharafutdinov, and I. E. Vnukov, Diffraction of real and virtual photons in a pyrolytic graphite crystal as source of intensive quasimonochromatic X-ray beam, Nucl. Instrum. Methods Phys. Res., Sect. B 201, 276 (2003).

[10] A. N. Baldin, I. E. Vnukov, B. N. Kalinin, and E. A. Karataeva, About real photon diffraction contribution into measured PXR spectrum in perfect crystals, J. Surf. Invest.: X-ray, Synchrotron Neutron Tech. 4, 72 (2006) (in Russian).

[11] R. Fulton, J. Haggerty, R. Jared, R. Jones, P. Kadyk, C. Field, W. Kozanecki, and W. Koska, A high resolution wire scanner for micron-size profile measurements at the SLC, Nucl. Instrum. Methods Phys. Res., Sect. A 274, 37 (1989).

[12] H. Hayano, in Proceedings of the 20th International Linac Conference, LINAC-2000, Monterey, CA, 2000 (SLAC, Menlo Park, CA, 2000), p. 146.

[13] M. Harrison, R. Agustsson, T. Hodgetts, A. Murokh, M. Ruelas, and P.S. Chang, in Proceedings of the 35th International Free-Electron Laser Conference, New York, 2013 (JACoW, Geneva, 2013), p. 276.

[14] S. A. Laktionova, O. O. Pligina, M. A. Sidnin, and I. E. Vnukov, Influence of real photons diffraction contribution on parametric X-ray observed characteristics, J. Phys. Conf. Ser. 517, 012020 (2014).

[15] H. Nitta, Kinematical theory of parametric X-ray radiation, Phys. Lett. A 158, 270 (1991).

[16] R. James, The Optical Principles of the Diffraction of $X$-rays (Bell, London, 1958).

[17] Z. G. Pinsker, Dynamical Scattering of X-Rays in Crystals (Springer, Berlin, 1984).

[18] Yu. A. Goponov, S. A. Laktionova, M. A. Sidnin, and I. E. Vnukov, Ratio of the contributions real and virtual photons diffraction in thin perfect crystals. Comparison of calculation and experiment, Nucl. Instrum. Methods Phys. Res., Sect. B 402, 92 (2017).

[19] Y. Takabayashi and A. V. Shchagin, Observation of parametric X-ray radiation by an imaging plate, Nucl. Instrum. Methods Phys. Res., Sect. B 278, 78 (2012).
[20] Y. Takabayashi, K. B. Korotchenko, Yu. L. Pivovarov, and T. A. Tukhfatullin, Angular distributions of parametric $\mathrm{X}$-ray radiation from a diamond crystal, Nucl. Instrum. Methods Phys. Res., Sect. B 402, 79 (2017).

[21] I. E. Vnukov, Y. A. Goponov, M. A. Sidnin, R. A. Shatokhin, K. Sumitani, and Y. Takabayashi, Measuring the Transverse Sizes of an Electron Beam by the Angular Distribution of the Coherent Radiation of Electrons in a Crystal, J. Surf. Invest.: X-Ray, Synchrotron Neutron Tech. 13, 515 (2019).

[22] Yu. A. Goponov, M. A. Sidnin, I. E. Vnukov, C. Behrens, G. Kube, W. Lauth, A. S. Gogolev, and A. P. Potylitsyn, Spatial distribution of PXR generated by $855 \mathrm{MeV}$ electrons: Comparison of simulation results with experimental data, Nucl. Instrum. Methods Phys. Res., Sect. B 402, 83 (2017).

[23] I. D. Feranchuk and A. V. Ivashin, Theoretical investigation of the parametric X-ray features, J. Phys. (Paris) 46, 1981 (1985).

[24] Yu. A. Goponov, S. A. Laktionova, O. O. Pligina, M. A. Sidnin, and I. E. Vnukov, Influence of real photon diffraction on parametric X-ray radiation angular distribution in thin perfect crystals, Nucl. Instrum. Methods Phys. Res., Sect. B 355, 150 (2015).

[25] Yu. A. Goponov, M. A. Sidnin, K. Sumitani, Y. Takabayashi, and I. E. Vnukov, Ultrarelativistic electron beam spatial size estimation from angular distribution emission in thin crystals, Nucl. Instrum. Methods Phys. Res., Sect. A 808, 71 (2016).

[26] I. Chaikovska, R. Chehab, X. Artru, and A. V. Shchagin, Characteristic, parametric, and diffracted transition X-ray radiation for observation of accelerated particle beam profile, Nucl. Instrum. Methods Phys. Res., Sect. B 402, 75 (2017).

[27] A. P. Potylitsin and V. A. Verzilov, Parametric X-rays and transition-diffracted radiation in crystal stacks, Phys. Lett. A 209, 380 (1995).

[28] A. A. Babaev and A. S. Gogolev, Thermal damage at short electron bunches passage through a thin target, J. Phys. Conf. Ser. 732, 012030 (2016). 